\title{
A Mixed Methods Investigation of Unmet Needs And Psychological Wellbeing During Active Surveillance For Prostate Cancer
}

Megan Mclntosh ( $\square$ megan.mcintosh@adelaide.edu.au )

University of Adelaide https://orcid.org/0000-0003-0933-6718

\section{Camille E Short}

University of Melbourne Department of Public Health: The University of Melbourne School of Population and Global Health

Michael O'Callaghan

Flinders University

\section{Andrew D Vincent}

The University of Adelaide - North Terrace Campus: The University of Adelaide

\section{Daniel A Galvão}

Edith Cowan University - Joondalup Campus: Edith Cowan University

\section{Melissa J Opozda}

The University of Adelaide

\section{Research Article}

Keywords: Active Surveillance, Prostate Cancer, Supportive Care, Qualitative Study, Mixed Methods, Unmet Needs

Posted Date: December 28th, 2021

DOI: https://doi.org/10.21203/rs.3.rs-1084154/v1

License: (a) (1) This work is licensed under a Creative Commons Attribution 4.0 International License. Read Full License 


\section{Abstract}

Purpose: While prostate cancer survivors experience unmet supportive care needs (USCNs) after definitive treatments, no study has measured USCNs during active surveillance (AS). This mixed-methods study aimed to identify and explore the USCNs and psychological wellbeing of AS patients.

Methods: Patients 18+ years diagnosed with prostate cancer, who had been on AS for $\geq 6$ months, were invited to complete a survey measuring USCNs, general and prostate cancer specific anxiety, and depression. A purposefully selected subset was also interviewed to explore USCNs and how needs during AS were addressed. Semi-structured interviews were transcribed verbatim and thematically analysed.

Results: One hundred and three participants ( $n=47$ currently on AS, $n=54$ on/had subsequent treatment, $\mathrm{n}=2$ ceased all care) completed the survey, and 33 were also interviewed. Although most USCNs were considered low/moderate, $20 \%$ of participants reported high unmet informational needs around receiving information about monitoring and their test results. USCNs related to patient care (e.g., access to services in rural areas or after hours) and sexuality were also discussed in interviews. Anxiety, depression, and prostate cancer specific anxiety were generally very low. Fear of cancer progression/recurrence was the highest scoring prostate cancer specific worry and was frequently mentioned by interview participants.

Conclusions: While unmet needs, anxiety and depression were generally low, one in five patients during AS experience unmet needs in psychological, physical, patient care, information, and sexual domains. Health professionals should be aware of common USCNs and offer appropriate support to address potential needs.

\subsection{Introduction}

\subsubsection{Background}

Active surveillance (AS) is the recommended treatment option for people with low risk prostate cancer [1]. It involves routine surveillance of biological markers of the disease through the use of prostate specific antigen (PSA) testing, digital rectal examinations, biopsies, and magnetic-resonance imaging (MRI) [1]. Transition to treatment is recommended upon identification of disease progression that constitutes reclassification [2]. Despite these recommendations and increased use of AS worldwide [2], there is no global consensus for AS initiation criteria, best practice for surveillance/follow-up, or triggers for intervention. As such, uptake and protocols vary greatly across clinics and countries [3-5]. This lack of consistency and consensus for best practice may impact patient's experiences, needs, and adherence during AS.

Approximately $12 \%$ of patients who begin AS will discontinue without evidence of disease progression, for largely unknown reasons [6], though factors such as anxiety, fearing mortality, and pressure from family/friends may contribute [4, 7]. Approximately $4-17 \%$ and $6-8 \%$ of patients on AS report generalised anxiety and depression, which is similar to rates among those who have definitive treatment [8-10]. 
However, much of this research has been conducted on samples recruited from cancer centres which specialise in AS management. Research which has recruited from a wider population has indicated that patients on AS consistently report high scores on anxiety and depression measures, which may actually increase over time [11]. Fear of progression may be a particular concern, as it is commonly reported in patients on AS [12-15] and is higher in patients on AS than in those who have undergone definitive treatment [16]. Persistent fear of progression during AS and ongoing anxiety may lead patients to discontinue AS prematurely to receive definitive treatments in an attempt to gain control of their health and disease [11].

Unmet supportive care needs may also contribute to discontinuation of AS, either directly or indirectly by negatively impacting psychological wellbeing [17-19]. A recent systematic review [20] suggests that patients on AS may experience a variety of unmet needs, most commonly related to inadequate and/or inconsistent information. However, only eight studies (five qualitative and three cross-sectional) were included in the review and the cross-sectional studies did not assess USCNs using a validated measure [20], such as the Supportive Care Needs Survey [21]. Just three of the studies had a primary aim of identifying unmet supportive care needs in patients on AS. Therefore, USCNs experienced by patients on AS, and their magnitude, are largely unknown, as is the extent of and ways in which unmet needs influence discontinuation of AS.

Understanding patient's experiences and needs during AS is essential for targeting support, to inform future research on issues faced by AS patients, and potentially improve compliance to AS management when appropriate.

\subsubsection{Aims}

This mixed methods study aimed to measure and explore the USCNs and psychological wellbeing of patients currently or who had previously been on AS after a prostate cancer diagnosis. A mixed methods approach to this study was considered important given the limited research to date and to allow potential identification of unmet needs not included in validated measures. Additionally, it may help us explore the methods patients have utilised to address unmet needs and which of these methods patients felt were successful.

\subsection{Methodology}

\subsubsection{Sample}

Participants were recruited as part of a larger case-control study designed to examine social-ecological factors associated with discontinuing AS [22]. The larger study, in which we had intended to recruit from multiple states in Australia, was paused due to COVID-19. Quantitative data collection from South Australia was completed prior to COVID-19. As such, this sub-study includes cross-sectional data from the South Australian participants. 
Eligible participants were identified and recruited through the South Australian Prostate Cancer Clinical Outcomes Collaborative (SA-PCCOC), which captures $90 \%$ of all diagnosed prostate cancer survivors in the state [23]. All eligible cases (individuals who had left AS and received definitive treatment without evidence of significant disease progression), and four matched controls (individuals still on active surveillance or who had received definitive treatment following adverse risk reappraisal, as clinically recommended) were invited to participate in the study $(\mathrm{N}=270)$ based on a sample size calculation for the main study.

Eligibility criteria for the survey were: diagnosed with prostate cancer between January 2014 and October 2019, commenced AS after diagnosis, on AS for at least six months (could still be on AS or since received further treatment), $18+$ years old. Patients were deemed ineligible if insufficient registry data was available to determine their treatment(s). Participants who completed the survey and provided their contact information on the consent form were considered for interviews and were selected using purposeful sampling [24]. Predetermined selection criteria were based on responses to the survey in regard to anxiety, depression, prostate specific anxiety, USCNs, rural/regional location, and reasons for or considering AS discontinuation (see supplementary material). Ability to participate in a telephone interview in English was also required.

\subsubsection{Procedure}

Ethics approval was obtained from Southern Adelaide Local Health Network (HREC/19/SAC/88).

\subsubsection{Survey}

They survey could be completed on paper, online, or via phone. Following eligibility screening, two pilot tests on the survey were conducted. The first assessed survey readability. Feedback was provided by four prostate cancer patients and two health professionals and adjustments to the survey were made accordingly. The second pilot test assessed response rate in a random sub-sample $(n=32)$ prior to the main mail-out. Six completed surveys (18.8\%) were returned. Given this low rate, changes were made in line with established methods for increasing response rates (i.e., use of a priming letter and unconditional monetary incentive) [25]. The remaining eligible participants $(n=238)$ were mailed the priming letter and one week later, the survey package (containing information sheet, consent form, survey, reply-paid envelope, and unconditional incentive). If no response was received after two weeks, a reminder letter was mailed. Participant demographics, clinical data, and treatment information were retrieved from SAPCCOC.

\subsubsection{Interviews}

Interviews were semi-structured and aimed to explore experiences on AS, USCNs, and psychological wellbeing (anxiety and depression) during AS (see supplementary material for interview guide). Participants were interviewed via phone, and interviews were audio recorded and transcribed verbatim. 
Interviews lasted on average 31 minutes (range 15-63 minutes). We invited all participants who met sampling criteria; as per Braun and Clarke's recommendations [26], thematic saturation was not considered the primary aim of recruitment.

\subsubsection{Outcome measures}

Validated measures (see Table 1) were used to assess USCNs, general and prostate cancer-specific anxiety, and depression. Demographic information was also assessed using standard items. Additional measures were included in the survey to assess potential predictors of adherence to AS (e.g., physical activity, coping strategies) and will be reported elsewhere.

Table 1

Survey Measures

\begin{tabular}{|c|c|c|}
\hline Variable & Measure & Description and scoring \\
\hline $\begin{array}{l}\text { Unmet } \\
\text { supportive } \\
\text { care needs }\end{array}$ & $\begin{array}{l}\text { Supportive } \\
\text { Care Needs } \\
\text { Survey - } \\
\text { Short } \\
\text { Form(Boyes } \\
\text { et al., 2009). }\end{array}$ & $\begin{array}{l}\text { This measure contains } 34 \text { items to assess unmet needs across five } \\
\text { domains: Physical \& Daily Living, Psychological, Health System \& } \\
\text { Information, Patient Care \& Support, and Sexuality. Items are } \\
\text { measured on a revised, validated(Schofield et al., 2012) 4-point Likert } \\
\text { Scale, where } 1=\text { no need, } 2=\text { low need, } 3=\text { moderate need, } 4=\text { =high need. }\end{array}$ \\
\hline $\begin{array}{l}\text { Anxiety } \\
\text { and } \\
\text { depression }\end{array}$ & $\begin{array}{l}\text { Patient Health } \\
\text { Questionnaire } \\
\text { for } \\
\text { Depression } \\
\text { and Anxiety } \\
\text { (Kroenke et } \\
\text { al., 2009). }\end{array}$ & $\begin{array}{l}\text { Assesses anxiety and depression using } 4 \text { items (two each for anxiety } \\
\text { and depression), across a } 4 \text {-point Likert scale }(0=\text { not at all; } 5=\text { nearly } \\
\text { every day). Total scores in each domain of } 3 \text { or above indicate } \\
\text { clinical threshold levels of anxiety/depression. }\end{array}$ \\
\hline $\begin{array}{l}\text { Prostate } \\
\text { cancer- } \\
\text { specific } \\
\text { anxiety }\end{array}$ & $\begin{array}{l}\text { Memorial } \\
\text { Anxiety Scale } \\
\text { for Prostate } \\
\text { Cancer(Roth } \\
\text { et al., 2003). }\end{array}$ & $\begin{array}{l}\text { Includes three sub-scales (general prostate cancer anxiety, anxiety } \\
\text { related to PSA testing, and fear of recurrence/progression), with items } \\
\text { measured across a } 5 \text {-point Likert scale }(0=\text { not at all; } 5=\text { nearly every } \\
\text { day). }\end{array}$ \\
\hline
\end{tabular}

\subsubsection{Analysis}

\subsubsection{Quantitative analyses}

Descriptive statistics for demographics, USCNs, anxiety, depression, and prostate cancer-specific anxiety were calculated in R [27]. In analysis, USCN categories were collapsed into none/low need and moderate/high need.

\subsubsection{Qualitative analyses}

Transcripts were analysed using Braun and Clarke's reflexive thematic analysis method [26]. This involves six key steps: familiarisation, coding, creating themes, revising themes, defining themes, and writing. Authors MM and MJO independently read and coded all transcripts. Both MM and MJO have experience in qualitative research and thematic analysis. MM and MJO discussed codes, and then all transcripts 
were re-analysed by MM to ensure all data was considered. Themes were then created, revised, and formally defined. Finally, themes were summarised with quotes from the transcripts, and refined by all authors.

\subsection{Quantitative Results 1.3.1 Participants}

One hundred and three participants returned a completed survey (38\% response rate). Of these, fortyseven were currently on AS, and fifty-four had received further treatment. Two participants indicated they had ceased all treatment and follow-up care. Most participants were married (83\%), retired (64\%), had completed post-secondary education (70\%), and lived in a major city (71\%; Table 2$)$. 
Table 2

Demographics

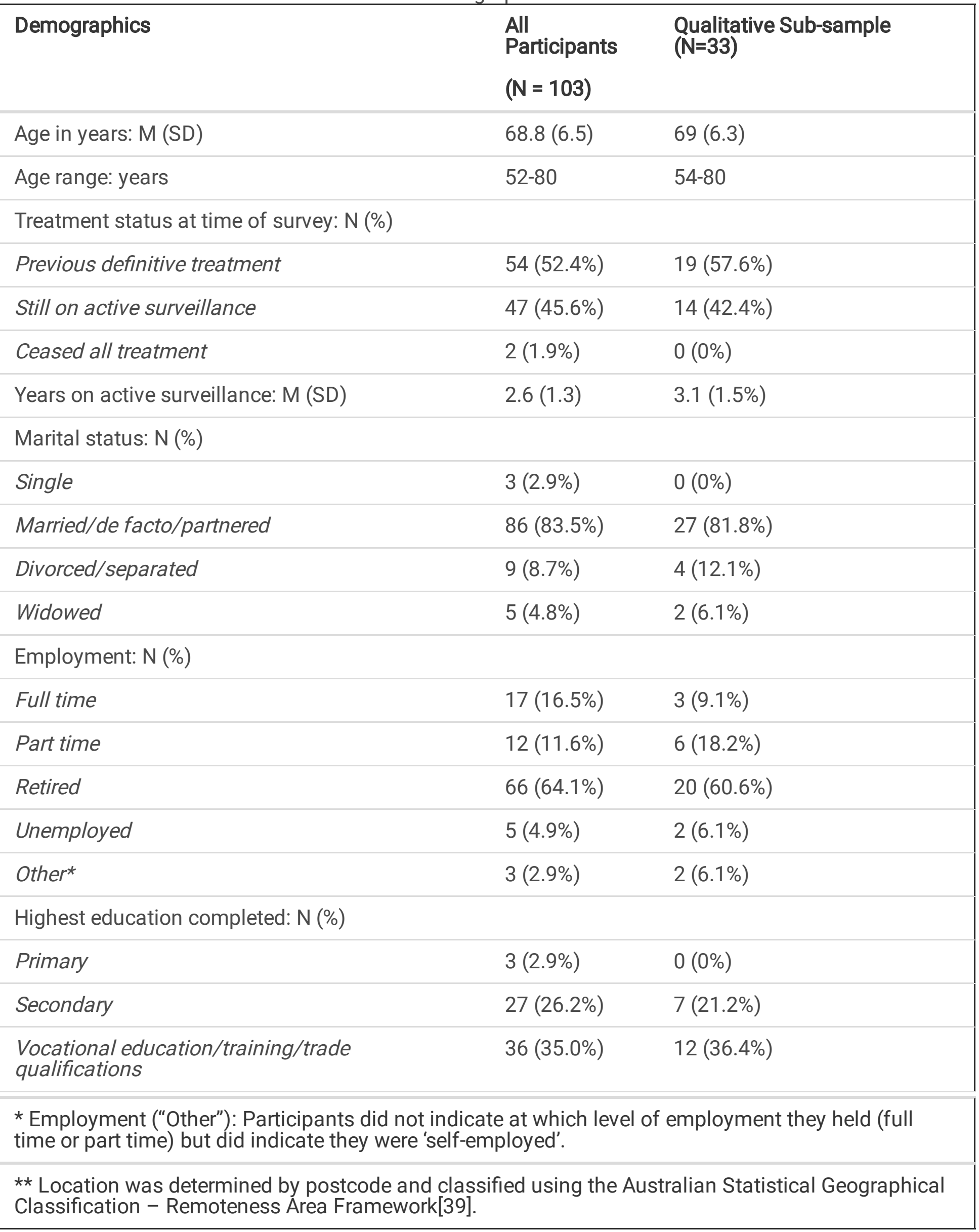




\begin{tabular}{|lll|}
\hline Demographics & $\begin{array}{l}\text { All } \\
\text { Participants } \\
(\mathbf{N}=103)\end{array}$ & $\begin{array}{l}\text { Qualitative Sub-sample } \\
(\mathbf{N}=33)\end{array}$ \\
\hline University & $36(35.0 \%)$ & $14(42.4 \%)$ \\
\hline Location**: N (\%) & & \\
\hline Very remote & $0(0 \%)$ & $0(0 \%)$ \\
\hline Remote & $5(4.9 \%)$ & $3(9.1 \%)$ \\
\hline Outer regional & $11(10.7 \%)$ & $6(18.2 \%)$ \\
\hline $\begin{array}{l}\text { Inner regional } \\
\text { Major city }\end{array}$ & $14(13.6 \%)$ & $3(9.1 \%)$ \\
\hline $\begin{array}{l}\text { * Employment ("Other"): Participants did not indicate at which level of employment they held (full } \\
\text { time or part time) but did indicate they were 'self-employed'. }\end{array}$ \\
\hline $\begin{array}{l}\text { ** Location was determined by postcode and classified using the Australian Statistical Geographical } \\
\text { Classification - Remoteness Area Framework[39]. }\end{array}$ \\
\hline
\end{tabular}

\subsubsection{Unmet supportive care needs}

An overview of the USCNs by domain is presented in Table 3. Moderate-high unmet needs were most common in the Health System and Information domain (e.g., being given written information about care, being informed of treatment side effects, informed of tests and monitoring results, provision of information and support from hospital staff), and Sexuality domains (e.g., changes in sexual feelings, relationships, and receiving sexual health information). Over one-fifth of participants (21.6\%) reported moderate-high unmet needs regarding fear of the cancer spreading (Psychological domain). Very few participants reported moderate-high unmet needs in the Physical and Daily Living and the Patient Care and Support domains. A complete summary of needs is presented in Table 4. 
Table 3

Scores for Anxiety, Depression and Supportive Care Needs

\begin{tabular}{|c|c|c|c|c|c|c|}
\hline \multirow[t]{2}{*}{ Measure / Domain } & \multicolumn{2}{|c|}{$\begin{array}{l}\text { All Participants } \\
(\mathrm{N}=103)\end{array}$} & \multicolumn{2}{|c|}{$\begin{array}{l}\text { Still on Active } \\
\text { Surveillance }(\mathrm{N}=47)\end{array}$} & \multicolumn{2}{|c|}{$\begin{array}{l}\text { Underwent Definitive } \\
\text { Treatment }(\mathrm{N}=54)\end{array}$} \\
\hline & $M(S D)$ & Range & $M(S D)$ & Range & $M(S D)$ & Range \\
\hline \multicolumn{7}{|c|}{$\begin{array}{l}\text { Anxiety and Depression } \\
\text { (PHQ-4) }\end{array}$} \\
\hline Anxiety & $\begin{array}{l}0.6 \\
(1.1)\end{array}$ & $0-5$ & $0.5(0.9)$ & $0-4$ & $0.6(1.2)$ & $0-5$ \\
\hline Depression & $\begin{array}{l}0.6 \\
(1.1)\end{array}$ & $0-6$ & $0.5(0.9)$ & $0-4$ & $0.6(0.3)$ & $0-6$ \\
\hline \multicolumn{7}{|c|}{$\begin{array}{l}\text { Supportive Care Needs } \\
\text { (SCNS) }\end{array}$} \\
\hline Physical & $\begin{array}{l}1.2 \\
(0.3)\end{array}$ & $\begin{array}{l}1- \\
2.4\end{array}$ & $1.1(0.3)$ & $1-2.4$ & $1.2(0.3)$ & $1-2.4$ \\
\hline Psychological & $\begin{array}{l}1.6 \\
(0.6)\end{array}$ & $\begin{array}{l}1- \\
3.3\end{array}$ & $1.5(0.6)$ & $1-3.3$ & $1.6(0.6)$ & $1-3.1$ \\
\hline Sexual & $\begin{array}{l}1.8 \\
(0.9)\end{array}$ & $1-4$ & $1.5(0.8)$ & $1-4$ & $1.9(0.9)$ & $1-4$ \\
\hline Care & $\begin{array}{l}1.3 \\
(0.5)\end{array}$ & $\begin{array}{l}1-\overline{6} \\
3.6\end{array}$ & $1.2(0.4)$ & $1-2.2$ & $1.4(0.5)$ & $1-3.6$ \\
\hline Informational & $\begin{array}{l}1.8 \\
(0.9)\end{array}$ & $1-4$ & $1.6(0.8)$ & $1-3.6$ & $2.1(0.9)$ & $1-4$ \\
\hline \multicolumn{7}{|l|}{$\begin{array}{l}\text { Prostate Cancer } \\
\text { Anxiety (MAXPC) }\end{array}$} \\
\hline Prostate cancer & $\begin{array}{l}0.6 \\
(0.6)\end{array}$ & $\begin{array}{l}0- \\
2.8\end{array}$ & $0.6(0.6)$ & $0-2$ & $0.7(0.7)$ & $1-2.3$ \\
\hline$P S A$ & $\begin{array}{l}0.1 \\
(0.4)\end{array}$ & $0-3$ & $0.1(0.3)$ & $0-1.3$ & $0.1(0.5)$ & $0-3$ \\
\hline Recurrence & $\begin{array}{l}0.7 \\
(0.7)\end{array}$ & $0-3$ & $0.7(0.7)$ & $0-3$ & $0.7(0.7)$ & $0-2.8$ \\
\hline
\end{tabular}


Table 4

Unmet Supportive Care Needs Experienced During Active Surveillance

Unmet Need
Total None/Low

$\mathrm{N}$

need

$\mathrm{N} \quad \% \quad \mathrm{~N} \quad \%$

Moderate/High

need

$\%$

Physical \& Daily Living

Lack of energy/tiredness

$\begin{array}{llll}101 & 94 & 93.1 & 7\end{array}$

6.9

Not being able to do things you used to do

10298

Pain

102

99

96.1

4

3.9

Work around the home

$102 \quad 101$

97.1

3

2.9

Feeling unwell a lot of the time

101

101

99.0

1

1.0

Psychological

Fears about the cancer spreading

102

Worry that the results of treatment are beyond your control

Concerns about the worries of those close to you

Uncertainty about the future

Learning to feel in control of your situation

Keeping a positive outlook

Feelings about death and dying

Feeling down or depressed

Anxiety

Feelings of sadness

Sexuality

Changes in sexual feelings

$\begin{array}{lllll}102 & 77 & 75.5 & 25 & 24.5\end{array}$

Changes in your sexual relationships

99

Being given information about sexual relationships

Patient Care \& Support

Reassurance by medical staff that the way you feel is normal

More choice about which cancer specialist you see 


\begin{tabular}{|c|c|c|c|c|c|}
\hline \multirow{2}{*}{$\begin{array}{l}\text { Unmet Need } \\
\text { Hospital staff acknowledging, and showing sensitivity to, } \\
\text { your feelings and emotional needs }\end{array}$} & \multirow{2}{*}{$\begin{array}{l}\text { Total } \\
\text { N } \\
98\end{array}$} & \multicolumn{2}{|c|}{$\begin{array}{l}\text { None/Low } \\
\text { need }\end{array}$} & \multicolumn{2}{|c|}{$\begin{array}{l}\text { Moderate/High } \\
\text { need }\end{array}$} \\
\hline & & 92 & 93.9 & 6 & 6.1 \\
\hline More choice about which hospital you attend & 100 & 94 & 94.0 & 6 & 6.0 \\
\hline Hospital staff attending promptly to your physical needs & 99 & 94 & 94.9 & 5 & 5.1 \\
\hline \multicolumn{6}{|l|}{ Health System \& Information } \\
\hline Being informed about the monitoring of the cancer & 100 & 61 & 61.0 & 39 & 39.0 \\
\hline Being informed about your test results as soon as feasible & 100 & 64 & 64.0 & 36 & 36.0 \\
\hline $\begin{array}{l}\text { Being adequately informed about the benefits and side- } \\
\text { effects of treatments before you choose to have them }\end{array}$ & 99 & 68 & 68.7 & 31 & 31.3 \\
\hline $\begin{array}{l}\text { Being informed about things you can do to help yourself } \\
\text { to get well }\end{array}$ & 100 & 69 & 69.0 & 31 & 31.0 \\
\hline $\begin{array}{l}\text { Having one member of hospital staff with whom you can } \\
\text { talk to about all aspects of your condition, treatment, and } \\
\text { follow up }\end{array}$ & 100 & 70 & 70.0 & 30 & 30.0 \\
\hline Being treated like a person not just another case & 99 & 70 & 70.7 & 29 & 29.3 \\
\hline $\begin{array}{l}\text { Being treated in a hospital or clinic that is as physically } \\
\text { pleasant as possible }\end{array}$ & 99 & 70 & 70.7 & 29 & 29.3 \\
\hline $\begin{array}{l}\text { Being given written information about the important } \\
\text { aspects of your care }\end{array}$ & 100 & 80 & 80.0 & 20 & 20.0 \\
\hline $\begin{array}{l}\text { Being given information (written, diagrams, drawings) } \\
\text { about aspects of managing your illness and any side } \\
\text { effects at home }\end{array}$ & 100 & 80 & 80.0 & 20 & 20.0 \\
\hline $\begin{array}{l}\text { Being given explanations of those tests for which you } \\
\text { would like explanations }\end{array}$ & 100 & 81 & 81.0 & 19 & 19.0 \\
\hline $\begin{array}{l}\text { Having access to professional counselling (e.g., } \\
\text { psychologist, social worker, counsellor, nurse specialist) if } \\
\text { you, family, or friends need it }\end{array}$ & 100 & 81 & 81.0 & 19 & 19.0 \\
\hline
\end{tabular}

\subsubsection{Psychological wellbeing}

Anxiety, depression, and prostate-specific anxiety were minimal. Only four (3.9\%) and five (4.9\%) participants met clinical thresholds for anxiety and depression, respectively (Table 3).

\subsection{Qualitative Results}

\subsubsection{Sample}

Thirty-three of the 37 eligible participants were interviewed ( $N=4$ could not be contacted or declined to participate). At the interview, 14 were still on AS and 19 had received further treatment since being on AS. 
Most were married (82\%), retired (61\%), completed post-secondary education (79\%), and lived in a major city (64\%), see Table 2. Nine (27\%) participants lived in an outer regional or remote area, four $(12 \%)$ met clinical thresholds for anxiety and/or depression, five (15\%) reported experiencing prostate-specific anxiety (in at least one of the subscales), and 13 (39\%) reported a moderate-high unmet need in at least one domain.

\subsubsection{Overview of themes}

Participants generally reported minimal USCNs and anxiety regarding their prostate cancer. Three overarching themes were identified. The first, "Unmet needs on AS", explores the unmet needs discussed by participants, and includes four sub-themes of specific unmet needs identified. The second theme, "How needs were addressed during AS", details the methods participants used to address needs and why they did not feel they experienced unmet needs after their cancer diagnosis. The final theme, "Hindsight is 20/20", explores the perspectives of participants who had since received definitive treatments on their unmet needs during AS. Illustrative quotes relevant to each theme/subtheme are presented in Table 5. 
Table 5

Illustrative Quotes for each theme/subtheme

\section{Unmet needs of patients during AS}

Informational Needs

- "If urology showed us models that showed prostates in various stages and that sort of thing that would probably help people to understand what's, what's being done, and why it's being done and how it's being done and so forth" Participant 10433, Active Surveillance

- "No, I didn't get a chance to talk to somebody, you know, especially in my community. No, no one I know who has got cancer, prostate cancer. And the issue they have, you know, we still have that stigma. Not to talk about it... So that lack of awareness in my [ethnicity] community is still there" Participant 9160, Definitive Treatment/s

Physical and Sexuality Needs

- "And I think I raised it with a GP, but my GPs at that time were ladies and whether they were reluctant to do anything to take that up, or whether they just didn't think it was very important. That has become more of a significant issue for me in more recent years. I have to say, but having said that, my wife has very severe back pain. And is, you know, there really wouldn't be much opportunity for that side of life anyway." Participant 13235, Definitive Treatment/s

Patient Care and Support Needs

- "Maybe a little bit more time, perhaps with the actual urologist maybe would be good or, or one that speaks, and I don't mean this badly, but one who's perhaps a little bit more GP orientated, a little bit better bedside manner, rather than just the medical side of things."Participant 14695, Active Surveillance

- "Oh yeah. That was really bad. And then if they want to do anything for country people, it would be to organise biopsies to be done in regional, country hospitals because it's not rocket science really." Participant 9107, Active Surveillance

Psychological Needs and Anxiety

- "Then the worry comes at the time that the numbers you know, when you keep going back to your PSA tests, and to see the results, you're hoping like hell, the numbers are gonna work out to be right, you know?"Participant 16058, Definitive treatment/s

- "Um, I won't say anxiety, but the uncertainty was annoying. And this goes back to this grey area I keep talking about. Beside that, just that uncertainty... you couldn't get the degree of facts-based support that I would have been, you know, that I'm entirely comfortable with. So that causes some anxiety." Participant 15132, Definitive Treatment/s

How needs were addressed during AS 


\section{Unmet needs of patients during AS}

- "Mostly I relied on the discussions with the doctor himself, or herself... The communication between them provided everything I needed really. Basically, it was, there was nothing that I wasn't unclear about that, or had concerns about, or any needs that were unmet." Participant 13959, Definitive Treatment/s

- "Pretty private dude. And I have my connections, friends and family who are, in in the systems and medical business side. It wasn't like I was out here by myself trying to work out what to do next. I had pretty good information. I didn't feel the need for anything other than my family support" Participant 9107, Active Surveillance

- "It's very important thing, it's a lot of worry off your mind... one thing I didn't have to worry about was funding for all that, all the, the treatment. You know, for either biopsy or the proctectomy, and the follow up treatments, all those things. All that was funded by DVA. So I was very, very fortunate that that happened." Participant 16058, Definitive treatment/s

- "But there hasn't been any sort of anxiety about that as such. At all, you know, when the test comes up, I've just been taking it in my stride. Because I think I trust the urologist." Participant 10421, Active Surveillance

\section{Hindsight is $20 / 20$}

- "I don't think I was shocked by not having [a referral to a prostate cancer nurse or physiotherapist]. But I think that there would be value, in retrospect, in having a service like that offered for men and their partners." Participant 13235, Definitive treatment/s

\subsubsection{Unmet needs of patients during AS}

\subsubsection{Informational needs}

Some participants identified topics they would have liked more information on during AS. One participant expressed a desire for more information and visual depiction of the prostate and its functions.

Additionally, several participants expressed uncertainty about their understanding of test procedures (e.g., biopsy and PSA), their safety and efficacy, and their effect on their overall health.

Another participant described the lack of understanding of AS and prostate cancer treatment options in his (ethnic minority) culture. This participant explained needing to keep his diagnosis and treatment private due to the stigma associated with cancer in his culture and community. The participant reported a need for increased information dissemination about AS and prostate cancer to minority communities in Australia and overseas.

\subsubsection{Physical and sexuality needs}

Participants regularly mentioned that a physical difficulty, such as erectile dysfunction and urinary incontinence, was the first symptom investigated that led to their prostate cancer diagnosis. Most participants had spoken to their urologist and/or general practitioner (GP) regarding these issues, and whilst some experienced improvements, for others they continued throughout AS.

\subsubsection{Patient care and support needs}


A more holistic form of healthcare (addressing full-body health) within AS follow-up appointments was desired by several participants. Participants wanted care to address ongoing physical symptoms (such as incontinence or erectile dysfunction), and to check for other health issues in blood tests, not just PSA levels. These participants expressed wanting a doctor who was compassionate and considered their whole health, rather than just their prostate cancer.

Most rural participants felt their experience and needs during AS were not significantly affected by their location. However, many acknowledged that long-distance travel could be difficult due to urinary incontinence or pain after a biopsy. Several participants also mentioned that AS would be easier if they could have biopsies and MRIs in hospitals closer to home. Furthermore, accessing support services was reported as more difficult when living rurally, due to confidentiality concerns, work schedules, or distance.

\subsubsection{Psychological needs and anxiety}

Whilst most participants reported experiencing very little or no anxiety in regard to their cancer, some felt anxious or worried before their appointments while waiting for test results. Participants described worrying that their cancer had progressed and would require treatment, or in some cases, that the cancer had metastasised and it was too late for treatment.

Several participants described feeling uncertain about staying on AS due to the inability of doctors to predict outcomes. Participants expressed a desire to receive objective recommendations and information specific to their diagnosis and expected future outcomes. Sometimes this uncertainty and unclear future left participants feeling anxious and worried.

\subsubsection{How needs were addressed during AS}

Information and support regarding prostate cancer and AS provided by the primary prostate cancer doctor was regularly described as sufficient for the patient's needs. Many reported they did not feel the need to access additional support from practitioners such as psychologists, dietitians, or prostate cancer nurses. Participants often confirmed they would be comfortable asking for referrals for additional support if they desired it.

Many participants reported seeking additional information via "Dr. Google", research papers, prostate cancer-related programs on television, textbooks, and prostate cancer or cancer websites (e.g., Cancer Council). Others found it beneficial to speak to other prostate cancer survivors or friends who were also medical professionals to discuss their experiences, seek support, or ask questions, particularly regarding definitive treatments.

Several men in the study had been members of the Australian Defence Force and held a Department of Veteran Affairs (DVA) healthcare card, providing them with financial support for cancer treatment, among other healthcare benefits [28]. Participants explained that holding a DVA card improved their experience and reduced their USCNs as it enabled them to access cancer treatment and other support services for free or at a discounted rate. 
Many of the participants in the study reported experiencing very little to no anxiety or unmet psychological needs regarding their cancer, the AS protocol, or the future during AS. Participants attributed their comfortability and lack of anxiety/depression to having trust and a good relationship with their doctor, having adequate access to information and support, and because they experienced minimal or no physical AS symptoms. Maintaining a positive outlook and objectivity was also considered to aid in reduced anxiety and worry.

\subsubsection{Hindsight is $20 / 20$}

For participants no longer on AS, reflecting on their unmet needs during AS helped them identify areas which they, in hindsight, thought would have helped to improve their AS experience. This was especially the case for psychological support - several participants had begun seeing a psychologist after undergoing definitive treatment and wished they had done so sooner. Similarly, some participants felt they may have had fewer unmet needs post-definitive treatment if they had accessed health professionals, such as a dietician, prostate cancer nurse, exercise physiologist, or physiotherapist, whilst on AS.

\subsection{Discussion}

\subsection{Discussion}

Despite research demonstrating that $80 \%$ of patients experience USCNs after definitive prostate cancer treatments [29], and over 35\% experience at least one USCN 15 years post-diagnosis [30], no research has used validated measures to explore and identify the USCNs of patients on AS. Given the unique management style AS provides to patients diagnosed with low risk, localised prostate cancer (which is the most common prostate cancer diagnosis [31]), understanding the needs of these patients is imperative for providing them with adequate care, support, and information. This is the first study to identify and measure the USCNs of patients on AS using a validated measure and a mixed-methods design. Within our sample, USCNs were reported across all domains, with moderate-high needs most commonly in informational, sexual, and patient care needs. However, average unmet needs per domain were reflective of low-moderate levels. In addition, our sample reported low levels of anxiety (general and prostate cancer-specific) and depression. Ways in which healthcare practitioners might address the USCNs mentioned by participants are summarised in Table 6 . 
Table 6

Unmet Needs by Supportive Care Needs Domains \& Recommendations

Need domain Unmet need Recommendations to address unmet need

Health

System \& Information
Information on

Prostate cancer, diagnosis, treatment options

Information on prostate anatomy

Understanding test results and purpose of test

Awareness of Prostate cancer in minority communities
Continue with provision of verbal and hard-copy information dissemination, referrals to online information, and support groups. Ensure information is up to date.

Doctors to provide explicit information on prostate. Include this information on handouts.

Doctors to provide specific information on patients tests results \& purpose of tests, as well as resources for general overviews.

Health professionals to consider cultural influence on knowledge/preferences during appointments. Advocate for information dissemination by public health services about Prostate cancer screening \& treatment to minority communities.

Patient Care \& Support
Financial support to access services and support
Lobbying for wider Medicare and pharmaceutical benefit coverage of treatments related to prostate cancer. Encourage use of available services through Medicare, Department of Veterans, etc for increased support.

Social support

Doctors provide information on support groups. Provision of support groups for patients on active surveillance. Involve spouse/partner where applicable.

Difficulty accessing services due to rurality

Accessing holistic healthcare

Doctors to utilize telehealth where possible (especially for short check-up appointments) for rural patients.

Consideration of appointment times for rural patients who must travel.

Doctors/health professionals to utilise multi-disciplinary healthcare services and coordinate ongoing care with services.

Accessing out-ofhours services (for patients working full-time or shiftwork)

Increased provision of after-hours psychological, support, and healthcare services for patients in rural areas, working full time, or working shiftwork. Potential for online provision of services.

Health professionals to collaborate \& employ standardised AS protocols and explain this clearly to patients. Advocation for national \& global AS protocol guidelines.
Uncertainty of AS (is it safe?)
Doctor to specifically ask patients about sexual functioning \& effect on intimacy with partner - offer support/referral if desired.
Addressing erectile incontinence and other symptoms on intimacy with partner
Sexual 


\begin{tabular}{|lll|}
\hline Need domain & Unmet need & Recommendations to address unmet need \\
\hline Physical & $\begin{array}{l}\text { Urinary } \\
\text { incontinence and } \\
\text { erectile } \\
\text { dysfunction }\end{array}$ & $\begin{array}{l}\text { Doctor to routinely check for signs of existing issue. } \\
\text { Considerations for patients traveling long-distances. }\end{array}$ \\
\hline
\end{tabular}

Just over $20 \%$ of the sample reported moderate-high unmet needs in at least one item across information, sexuality, psychological, and patient care domains. Unmet informational needs were the most common; participants reported a desire for written information about AS care, treatment options and side effects, and receiving test results and explanations for these results. Similar unmet informational needs are often reported by prostate cancer patients after definitive treatments [29,32], and were the most common reported need in a systematic review on USCNs in patients on AS [20]. Several unique unmet informational needs were identified in our qualitative analysis. Unmet needs related to the desire for information specific to the role and functioning of the prostate and the ways in which it is affected by prostate cancer treatments and tests was mentioned by one participant. Another participant discussed unmet informational needs regarding the awareness of prostate cancer (i.e., treatment options including AS and definitive treatments) in his minority ethnicity community. In line with recent recommendations for practitioners working with prostate cancer survivors [33], these unmet informational needs may be addressed through an increased focus on disseminating information on men's health and prostate cancer to primary care providers, community workers, and prostate cancer survivors and their families, especially those living in rural/remote areas and people from culturally and linguistically diverse (CALD) backgrounds. Ensuring messaging is consistent, readily available, evidence-based, culturally appropriate, and takes into account existing health literacy and preferences for information is essential for reducing unmet informational needs [33].

Sexuality needs were the second most common moderate-high USCN by patients in the present study. Information on addressing sexual function and intimacy issues is a common unmet need for patients post-definitive treatment, given that erectile dysfunction is a common side effect of most prostate cancer treatments [19]. The present study has identified that, like patients after definitive prostate cancer treatment, patients on AS may also require information and support regarding their sexuality and sexual function. Similar again to USCNs seen after definitive treatments [29, 32], physical unmet needs relating to urinary incontinence were also reported by participants on AS. Health practitioners with patients on AS must ensure they provide information and support and routinely check-in with patients to identify if additional support or information regarding sexuality or physical function is required.

Finally, although generalised and prostate cancer-specific anxiety scores were generally low across participants, over $20 \%$ of participants reported fear of cancer progression as an unmet psychological need while on AS. Feelings of fear, worry, and uncertainty were also regularly discussed by participants in interviews. Participants described feeling concerned and fearful of the cancer spreading too quickly, negating their ability to cure the cancer with definitive treatments. Previous research has demonstrated that fear of progression and anxiety, whilst generally low and similar to levels reported by patients after definitive treatment, are experienced by patients on AS [8,34,35]. Psychological unmet needs and fear of 
progression may be combatted by reducing unmet informational needs (e.g., providing clear and consistent information about AS, test results, treatment options, and triggers for definitive treatment) and routinely discussing psychological wellbeing and providing referrals to psychological or other services when applicable. This is especially vital given that several participants in the present study reported difficulties in accessing support services due to their rural location or work schedules. Furthermore, many participants in the study who had since received definitive prostate cancer treatment shared that in retrospect, additional psychological support, access to a prostate cancer nurse, or referrals to a dietician or exercise physiologist may have been beneficial during AS to improve their experience and anxiety/fears.

\subsubsection{Limitations and future research}

Several limitations should be considered when interpreting these results. The original case-control study (which intended to recruit from multiple Australian states) was unable to proceed due to COVID-19 shutdowns. Consequently, our sample size was considerably lower than originally planned and prevented us from statistically investigating predictors of higher USCNs. Further research with larger samples is therefore required to understand the extent of USCNs in patients on AS both in Australia and worldwide. With no global AS protocol and wide variations in healthcare services and systems, research is also to determine whether unmet needs differ by location. While our findings suggest that patients from culturally and linguistically backgrounds may experience unique USCNs after prostate cancer diagnosis, as we only recruited participants who could communicate fluently in English, these results are limited, and further investigation is needed.

\subsubsection{Conclusions}

This cross-sectional mixed-methods study is the first to measure and explore the USCNs experienced by patients during AS using a validated measure and interviews. While unmet needs, anxiety and depression were generally low, one in five patients during AS experience unmet needs in psychological, physical, patient care, information, and sexual domains. To reduce unmet needs, including anxiety and fears of cancer progression, and potentially prevent AS discontinuation prematurely, it is essential that health professionals provide consistent and clear information, and enquire about their patient's needs and psychological wellbeing regularly.

\section{Declarations}

Funding: This study was funded by a Below the Belt research grant through the Australian and New Zealand Urogenital and Prostate Cancer Trials Group (ANZUP). Author MM was supported by an Australian Government Research Stipend during her PhD and received further support from well as the Freemasons Centre for Male Health and Wellbeing.

Conflicts of Interests: The authors declare there are no relevant financial or non-financial interests to disclose. 
Author Contributions: All authors contributed to the study conception and design. Material preparation, data collection, analysis, and the manuscript draft was primarily performed by Megan Mclntosh. Remaining authors provided critical feedback and assistance through all stages of the study (material preparation, data collection, analysis, and manuscript drafts). All authors read and approved the final manuscript.

Availability of data and material: Data will be made available on Figshare. Please contact the corresponding author.

Code availability: Not applicable.

Ethics Approval: Ethics approval for this project was obtained through the Southern Adelaide Local Health Network (SAHLN). Approval number: HREC/19/SAC/88.

Consent to participate: Informed consent was obtained from all individual participants.

Consent to publish: The authors confirm that all participants provided informed consent and consented to the use of their data (de-identified) being included in this manuscript.

\section{References}

1. Sanda MG, Cadeddu JA, Kirkby E, Chen RC, Crispino T, Fontanarosa J et al (2018) Clinically Localized Prostate Cancer: AUA/ASTRO/SUO Guideline. Part I: Risk Stratification, Shared Decision Making, and Care Options. J Urol 199(3):683-690

2. Bruinsma SM, Nieboer D, Roobol MJ, Bangma CH, Verbeek JFM, Gnanapragasam V et al (2021) RiskBased Selection for Active Surveillance: Results of the Movember Foundation's Global Action Plan Prostate Cancer Active Surveillance (GAP3) Initiative. J Urol 206(1):62-68

3. Kinsella N, Helleman J, Bruinsma S et al (2018) Active surveillance for prostate cancer: a systematic review of contemporary worldwide practices. Transl Androl Urol 7:83-97

4. Kinsella N, Stattin P, Cahill D et al (2018) Factors Influencing Men's Choice of and Adherence to Active Surveillance for Low-risk Prostate Cancer: A Mixed-method Systematic Review. Eur Urol 74(3):261-280

5. Walker CH, Marchetti KA, Singhal U, Morgan TM (2021) Active surveillance for prostate cancer: selection criteria, guidelines, and outcomes. World J Urol1-8. https://doi.org/10.1007/s00345-02103622-8

6. Van Hemelrijck M, Ji X, Helleman J et al (2018) Reasons for Discontinuing Active Surveillance: Assessment of 21 Centres in 12 Countries in the Movember GAP3 Consortium. Eur Urol 75(3):523531

7. Mclntosh M, Opozda M, Galvão DA et al (2019) Identifying the exercise-based support needs and exercise programme preferences among men with prostate cancer during active surveillance: $A$ qualitative study. Eur J Oncol Nurs 41:135-142 
8. Carter G, Clover K, Britton B et al (2015) Wellbeing during Active Surveillance for localised prostate cancer: A systematic review of psychological morbidity and quality of life. Cancer Treat Rev 41:4660

9. Bellardita L, Valdagni R, van Den Bergh R et al (2015) How Does Active Surveillance for Prostate Cancer Affect Quality of Life? A Systematic Review. Eur Urol 67:637-645

10. Ruane-Mcateer E, Porter S, Sullivan JM et al (2016) Active Surveillance for Favourable-Risk Prostate Cancer: Is there a Greater Psychological Impact than Previously Thought? A Systematic, Mixed Studies Literature Review. Psychooncology. https://doi.org/10.1002/pon.4311

11. Ruane-McAteer E, Porter S, O'Sullivan J et al (2019) Investigating the psychological impact of active surveillance or active treatment in newly diagnosed favorable-risk prostate cancer patients: A 9month longitudinal study.Psych Onc. https://doi.org/10.1002/pon.5161

12. Nilsson R, Næss-Andresen TF, Myklebust T et al (2021) Fear of Recurrence in Prostate Cancer Patients: A Cross-sectional Study After Radical Prostatectomy or Active Surveillance. Eur Urol Open Sci 25:44-51

13. Ervik B, Nordøy T, Asplund K (2010) Hit by waves-living with local advanced or localized prostate cancer treated with endocrine therapy or under active surveillance. Cancer Nurs 33:382

14. Hedestig O, Sandman P-O, Widmark A (2003) Living with untreated localized prostate cancer: A qualitative analysis of patient narratives. Cancer Nurs 26:55-60

15. Berger Z, Yeh J, Carter H, Pollack C (2014) Characteristics and Experiences of Patients with Localized Prostate Cancer Who Left an Active Surveillance Program. Patient 7:427-436

16. Parker PA, Davis JW, Latini DM et al (2016) Relationship between illness uncertainty, anxiety, fear of progression and quality of life in men with favourable risk prostate cancer undergoing active surveillance. BJU Int 117:469-477

17. Fitch MI (2008) Supportive care framework. Can Oncol Nurs J 18:6

18. Harrison JD, Young JM, Price MA et al (2009) What are the unmet supportive care needs of people with cancer? A systematic review. Support Care Cancer 17:1117-1128

19. Hamdy FC, Donovan JL, Lane JA et al (2016) 10-Year Outcomes after Monitoring, Surgery, or Radiotherapy for Localized Prostate Cancer. N Engl J Med 375:1415-1424

20. Mclntosh M, Opozda MJ, Evans $\mathrm{H}$ et al (2019) A systematic review of the unmet supportive care needs of men on active surveillance for prostate cancer. Psychooncology 28:2307-2322

21. Boyes A, Girgis A, Lecathelinais C (2009) Brief assessment of adult cancer patients' perceived needs: development and validation of the 34-item Supportive Care Needs Survey (SCNS-SF34. J Eval Clin Pract 15:602-606

22. Mclntosh M (2020) Why do men leave active surveillance? A case-control study examining factors contributing to non-adherence (protocol). In: ANZCTR. http://www.anzctr.org.au/Trial/Registration/TrialReview.aspx?id=379100\&isReview=true. Accessed 22 Mar 2021 
23. SA-PCCOC. https://www.prostatehealth.org.au/. Accessed 14 May 2021

24. Palinkas L, Horwitz S, Green C et al (2015) Adm Policy Ment Heal Ment Heal Serv Res 42:533544Purposeful Sampling for Qualitative Data Collection and Analysis in Mixed Method Implementation Research

25. Edwards PJ, Roberts I, Clarke MJ et al (2009) Methods to increase response to postal and electronic questionnaires. Cochrane Database Syst. Rev 3. DOI: ̈̈»¿10.1002/14651858.MR000008.pub4

26. Braun V, Clarke V, Hayfield N, Terry G (2019) Thematic analysis. In: Handbook of Research Methods in Health Social Sciences. Springer Singapore, pp 843-860

27. R Core Team (2019) R: A Language and Environment for Statistical Computing. ï»¿https://www.rproject.org

28. Veteran Card | Department of Veterans' Affairs. https://www.dva.gov.au/health-andtreatment/veteran-healthcare-cards/veteran-card. Accessed 29 Apr 2021

29. Paterson C, Robertson A, Smith A, Nabi G (2015) Identifying the unmet supportive care needs of men living with and beyond prostate cancer: A systematic review. Eur J Oncol nursing 19:405-418

30. Mazariego CG, Juraskova I, Campbell R, Smith DP (2020) Long-term unmet supportive care needs of prostate cancer survivors: 15-year follow-up from the NSW Prostate Cancer Care and Outcomes Study. Support Care Cancer 28:5511-5520

31. American Cancer Society Survival Rates for Prostate Cancer.

ï»¿https://www.cancer.org/cancer/prostate-cancer/detection-diagnosis-staging/survival-rates.html

32. King AJL, Evans M, Moore THM et al (2015) Prostate cancer and supportive care: a systematic review and qualitative synthesis of men's experiences and unmet needs. Eur $\mathrm{J}$ Cancer Care (Engl) 24:618-634

33. Dunn J, Green A, Ralph N et al (2020) Prostate Cancer Survivorship Essentials Framework: Guidelines for Practitioners.BJU Int. https://doi.org/10.1111/bju.15159

34. van den Bergh RCN, Essink-Bot ML, Roobol MJ et al (2010) Do Anxiety and Distress Increase During Active Surveillance for Low Risk Prostate. Cancer? J Urol 183:1786-1791

35. Latini DM, Hart SL, Knight SJ et al (2007) The Relationship Between Anxiety and Time to Treatment for Patients With Prostate Cancer on Surveillance. J Urol 178:826-832

\section{Supplementary Files}

This is a list of supplementary files associated with this preprint. Click to download.

- SupplementaryMaterial.docx 